\title{
7 Outlook
}

At the end of this thesis, possible enhancements of the proposed approaches as well as future research directions are discussed briefly.

All the approaches that have been proposed in this work are only applied to and evaluated on load spectrum data. However, this kind of automotive data has some disadvantages, as discussed in Chapter 2. It does, for example, not store any information about the time of occurrence of the transformed signal values of interest. Moreover, its quality is also dependent on the data resolution, e.g., on the number of intervals and the definition of each of them.

Hence, in a future work, it may be beneficial to compensate for this lack of information by enriching load spectrum data with other kinds of data such as the error memory data [99] of the vehicles. This may improve the provided classification results and allow to learn even more significant rules for predicting failure types of certain components.

Furthermore, each individual class or interval of a load spectrum has been used as a separate input variable in this thesis. Thereby, the information that is given in each class description, like the interval specification, is not exploited by the proposed approaches. Thus, in a future analysis some statistics that approximate, e.g., the central tendency and the dispersion of the distribution that is underlying each load spectrum may be calculated. Among these measures are the mean and the variance and so on. This is partly done in [40], but only for load spectrum data resulting from one-parameter counting methods. Moreover, Frisk et al. do not determine higher-order statistics, like the skewness and kurtosis, for each load spectrum, which may provide additional useful information.

Finally, modern telematics services will transmit the recorded load spectrum data of each vehicle to databases, owned by the OEM, in regular time intervals in the future, e.g., every week or month. This will offer new possibilities for analysing this kind of data. For example, the variation of the amount of growth of each load spectrum over time, i.e., the absolute or relative differences between load spectra of consecutive transfer dates, may provide useful information. As a consequence, there will be an urgent need for methods that allow to analyse time-series of load spectra in the future. 